REVIEW

\title{
How has research in the last five years changed my clinical practice?
}

\section{A Laing}

Arch Dis Child Fetal Neonatal Ed 2005;90:F364-F367. doi: 10.1136/adc.2004.064196

Although the practice of neonatology is increasingly evidence based, much of our assessment and treatment of newborn infants is still founded on experience, common sense, guesswork, and myth. Research may put a particular issue beyond dispute, but it often raises new questions. This review looks at the benefits of research in one area: temperature control.

Correspondence to: Dr Laing, Neonatal Unit, Simpson Centre for Reproductive Health, 51 Little France Crescent, Edinburgh EH16 4SU Scotland, UK; ian.laing@ luht.scot.nhs.uk

Accepted 16 March 2005
A lthough the practice of neonatology is increasingly evidence based, much of our assessment and treatment of newborn infants is still founded on experience, common sense, guesswork, and myth. A detailed study of a large, randomised, double blinded, controlled trial or a visit to the Cochrane database may be highly informative and can provide a secure platform on which to build safe clinical practice. For clinicians, however, there are many other influences that change their practice including an anecdote from the literature, a casual conversation with a respected colleague, an inspirational visiting lecturer, interaction with parents and other members of staff, and merely what happened the last time such a treatment was tried. In our determined efforts to find the best pathway for our patients, we are enriched by, or vulnerable to, the suggestions of those around us.

\section{RESEARCH}

Research may put a particular issue beyond dispute: we know that replacement surfactant improves the outcome of preterm infants with respiratory distress syndrome. But research often raises new questions: the administration of steroids can coax a baby with chronic lung disease from a ventilator, but what (if any) are the definitive indicators for starting treatment, when is its use safest for the infant, in what dose and for how long?

It is also important to recognise that research pathways that are incomplete may nevertheless influence clinical decisions pending emergence of the full picture. It is in this spirit that the following paragraphs are written. My personal reactions to recent research may differ from those of my colleagues. In practice, neonatologists are constantly choosing pathways, and often discover that these routes differ from those chosen by others. It is our hope that the destination of safety proves to be the same.
THE TASK

It is tempting for a commissioned author to review as many as possible of the recent changes in clinical practice and to identify the influences that brought about these changes. I have, for example, developed an increasing enthusiasm for prophylactic replacement surfactant in infants less than 29 weeks gestation at birth. In 2004, despite caring for 111 infants weighing less than $1500 \mathrm{~g}$ at birth, in the Simpson neonatal unit we inserted no chest drains in any infant in our care. No infant developed a pneumothorax on assisted ventilation; two small pneumothoraces resolved spontaneously and one moderate pneumothorax resolved after needle aspiration. Of course many other reasons for this could be advanced apart from our current use of natural surfactant, such as subtle changes in ventilation technique, increased use of continuous positive airways pressure, and perhaps good fortune. Rather than flit from one deeply held prejudice to another, in this review I will concentrate on one subject only, temperature control, in two very different situations.

\section{BRAIN COOLING IN ASPHYXIA}

In December 2003, at the Hot Topics in Neonatology meeting, Professor Peter Gluckman provided for his audience in Washington the first indications that cooling the brain of an asphyxiated infant may prove beneficial and that it seemed, in the hands of chosen centres, a relatively safe procedure on which to embark. One year later, further details have emerged. ${ }^{1}$ The questions multiply. ${ }^{1-3}$ How does one define the term asphyxia to identify the group of children who may benefit from cooling? How safe is safe? Is brain cooling preferable to total body cooling? Which temperature is best? When should cooling take place? How quickly or slowly should rewarming take place? Perhaps most challenging of all is the ethical conundrum of parental consent for randomised studies: if obtaining fully informed consent takes several hours, causing the window of opportunity to be missed, is it ethical to embark on cooling (as yet of unproven benefit) without obtaining such parental consent?

In the "cool-cap" study, 234 term infants with moderate or severe neonatal encephalopathy plus abnormal amplitude integrated electroencephalography (aEEG) were randomised to either head cooling for 72 hours within 6 hours of birth or conventional care. Rectal temperature of the brain cooled group was maintained at $34-35^{\circ} \mathrm{C}$. The primary outcome was death or severe disability at 18 months. There was no difference 
identified in acute adverse events. There was no overall significant difference in death and severe disability at 18 months. Nevertheless, the authors had predefined subgroups of infants with severe and less severe aEEG changes; brain cooling appeared to provide a significant benefit in the group with less severe aEEG changes.

Shankaran and coworkers ${ }^{2}$ have reported preliminary results of their multicentre trial of whole body cooling for hypoxic ischaemic encephalopathy. The trial studied infants of gestation $\geqslant 36$ weeks, age $<6$ hours, and with a cord $\mathrm{pH}$ or first hour of life $\mathrm{pH} \leqslant 7.0$, or a history of a perinatal event and a need for resuscitation at birth. From this group, 238 infants were identified with moderate or severe hypoxic ischaemic encephalopathy, of whom 208 were randomised into the trial. Of these, 102 were randomised to the hypothermia group and were cooled within 45 minutes to a target temperature of $33.5^{\circ} \mathrm{C}$ for 72 hours. The primary outcome of the study was death or severe disability at 18 months of age. The risk of death or serious adverse events did not seem to be different between the two groups. However, the risk of death and moderate/severe disability was $45 \%$ in the hypothermia group and $62 \%$ in the normothermia group, a result that just achieved statistical significance. The authors recognised that more information is required from longer term follow up, and they concluded that, within the parameters of the study, whole body cooling seemed a "safe" technique.

Meanwhile Eicher and coworkers ${ }^{3}{ }^{4}$ have published in 2005 studies examining efficacy and safety outcomes of using moderate body hypothermia. They report the results of a multicentre, randomised controlled pilot trial designed to study the effects of 48 hours of cooling to $33^{\circ} \mathrm{C}$. Inevitably their inclusion criteria differed in detail from those of the study of Shankaran et al, and most of the infants in their study were outborn. Thirty two infants were randomised to the hypothermia group, and 33 were in the normothermic group. Infants in the hypothermia group had significantly lower platelet counts even 36 hours after rewarming, and there was a significantly higher median prothrombin time on day 4 . There was also significantly more haematuria observed in the hypothermia group. The hypothermia group were also more likely to show profound bradycardia and require nitric oxide therapy for pulmonary hypertension, and they had an increased need for pressor support even 60 hours after rewarming. Despite these concerns, combining death and severe motor disability as the outcome measure showed significantly fewer bad outcomes in the hypothermia group.

\section{RESPONSE OF A CLINICIAN (1)}

How is a clinician to respond to these data in 2005? The coolcap study produced data that are promising for infants with less severe aEEG changes. In 2005, electronic beaming of data is increasingly widespread, and it should be possible for all intensive care units to obtain an aEEG tracing, transmit it to a UK interpretation base, and identify the infants who may receive neuroprotective benefit from brain cooling. In a small number of centres, the technique has not yet shown significant adverse effects, but it must be carefully monitored if units new to the implementation are to be involved. First do no harm. It is my firmly held view that all centres intending to use brain cooling in the coming years must be fully equipped and trained, and should embark on brain cooling only under strict protocols as part of a multicentre research programme. To do otherwise would be unethical.

The data on total body cooling are, in my opinion, more controversial. Should transient thrombocytopenia, coagulopathy, and haematuria be sufficient warning signs to be cautious of this unproven treatment? Is the hypotension and need for pressor support a real worry or might it be the effects of rewarming too quickly? On the positive side, in the study of Eicher and coworkers ${ }^{3}$ combining death and severe motor disability as an outcome measure appeared to show a significant benefit to the hypothermia group. But is the benefit due to the technique itself? Clearly the study could not be blinded. The clinicians and the parents knew whether an individual infant was in the hypothermia or normothermia group. Could there be some subtle influence working here? Parents of infants randomised to the active hypothermia group may have faith in the protective effect of the technique and therefore may be more reluctant to consent to withdrawal of active neonatal intensive care. More work needs to be carried out to explore the implications of the immediate adverse effects and most important of all the long term outcome of the two groups. As further studies progress, it will become clear to clinicians which subgroups of infants (if any) will benefit most from brain cooling. Neither Shankaran et $a l^{2}$ nor Eicher $e t a l^{3}{ }^{4}$ used aEEG as a possible predictor of infants who might respond to total body cooling, and so part of their studies are not comparable with the work of the cool-cap study group.'

Meanwhile, how has this impacted on my care of the small number of infants seen annually who are in this perilous situation? I reject the idea that all neonatal units that provide intensive care should embark on induced hypothermia for asphyxiated infants outwith a randomised controlled trial. Given the early adverse side effects identified in the most carefully controlled conditions, this may cause harm to some infants in our care. My view may well be revised in a small number of years, but in 2005 I believe it is reasonable to be cautious and to strive to prevent hyperthermia. In the neonatal intensive care unit in Edinburgh, we admit these unfortunate infants to open cots where we can ensure that their core temperature is maintained at $\leqslant 37^{\circ} \mathrm{C}$. It is possible that hyperthermia causes harm, and this we must avoid. It is also possible that hypothermia may be protective to some infants, and we await the proof that this is so and the accurate identification of the particular infants who may show clinical benefit. Meanwhile anxieties about short term adverse consequences shown by Eicher and coworkers ${ }^{3}$ must stay our hand, at least in terms of embarking on total body cooling.

\section{HYPOTHERMIA IN EXTREME PRETERM INFANTS}

One of the most influential neonatal studies of the last decade is the EPICure study ${ }^{5}$ from which have emerged many important observations about the outcomes of infants born less than 26 weeks gestation. In this prospective study of over 4000 births, multivariate analysis showed that an admission temperature of $<35^{\circ} \mathrm{C}$ was independently associated with death (odds ratio $0.58,95 \%$ confidence interval 0.39 to 0.85 , $\mathrm{p}=0.006$ ). In 2001, Oxley and Lyon ${ }^{6}$ reported the outcome of 116 infants born at less than 29 weeks gestation and whose temperature was recorded on admission; they found by stepwise multiple logistic regression that an admission temperature of $\leqslant 35^{\circ} \mathrm{C}$ was independently associated with death. Indeed, on multiple regression, only gestational age and admission temperature remained independently associated with death $(\mathrm{p}=0.02)$.

Meanwhile in 1999, Vohra and coworkers ${ }^{7}$ reported their study on the effectiveness of polyethylene wrap applied immediately at birth, without prior drying, to 59 infants $<32$ weeks gestation at birth. They noted an improvement by $1.9^{\circ} \mathrm{C}(\mathrm{p}<0.001)$ in the group of gestation $<28$ weeks, and they further commented that all five deaths in their study were in the group randomised to conventional care with no polyethylene wrap. Intriguingly this technique had first been described in 1971, ${ }^{8}$ albeit for infants weighing more than $2000 \mathrm{~g}$, but had not become a widely adopted practice in caring for preterm neonates. 
Since 2000, in Edinburgh, infants less than 29 weeks gestation have been placed immediately on delivery into a clean plastic bag. The baby slides easily in, provided that no skin drying has been used. Resuscitative procedures are readily carried out inside the bag with as little as possible of the head exposed. A woollen hat is used. Intubation, administration of surfactant, and fixation of the endotracheal tube (fig 1) are carried out while evaporative heat loss is minimised. Clinical inspection and auscultation are easily accomplished. Blankets are not put over the infant, so that the radiant heat warmer can warm the infant through the plastic bag. If vascular access is required, a small hole can be cut in the bag. On arrival in the neonatal unit, the infant remains in the bag during weighing and transfer to the prewarmed and humidified incubator. Only when the incubator shows the predicted neutral thermal environment and $80 \%$ humidity do the staff remove the bag. If lines or monitors are required before that moment, fixation can be achieved by cutting small holes in the plastic bag.

In 1992 the British Association of Perinatal Medicine/Royal College of Physicians (BAPM/RCP) set a standard of $36^{\circ} \mathrm{C}$ or more as an acceptable admission temperature for newborns. ${ }^{9}$ More recently the CESDI Project 27/28 studied details of the infants who died at 27-28 weeks gestation in England, Wales, and Northern Ireland during a two year period..$^{10}$ The temperature on admission failed to reach the standard in $73 \%$ of babies who died and 59\% of controls. The CESDI study added to the EPICure observation ${ }^{5}$ that temperature on admission is an independent variable in predicting death in extreme preterm babies. However, the CESDI report took a pessimistic view about whether the BAPM/RCP standard could be achieved and stated "the feasibility of achieving this standard must be questioned". In Edinburgh, however, using the plastic bag to minimise evaporative heat loss, the mean temperature on admission is $37(0.7)^{\circ} \mathrm{C}$ and no inborn extreme preterm babies have temperatures under $36^{\circ} \mathrm{C}$ on admission to the neonatal unit. ${ }^{11}$ Furthermore, Meyer and coworkers, ${ }^{12}$ using a similar technique, measured the admission temperature of babies less than $1000 \mathrm{~g}$ at birth and showed a similar improvement.

In December 2004, Vohra et $a l^{13}$ reported a randomised controlled trial of polyethylene occlusive skin wrapping in infants less that 28 weeks gestation at birth. Of 53 infants who completed the study, wrapped infants had an admission temperature of $36.5(0.8){ }^{\circ} \mathrm{C}$ compared with a control group who were dried conventionally at birth and whose admission temperature was $35.6(1.3)^{\circ} \mathrm{C}$. Two wrapped infants had an admission temperature on admission above $37.5^{\circ} \mathrm{C}$. It was not reported what the temperatures of the mothers were at delivery.

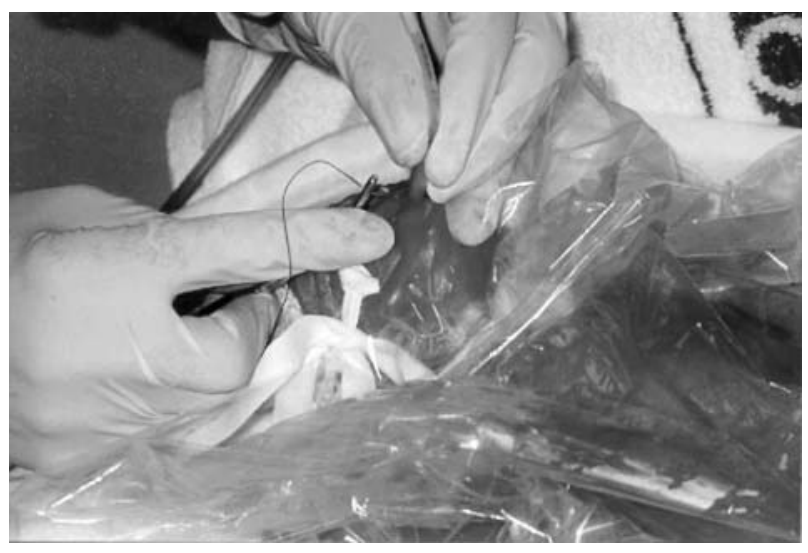

Figure 1 Fixation of an endotracheal tube in an extreme preterm infant, with the use of a woollen hat and bag to minimise evaporative heat loss.
RESPONSE OF A CLINICIAN (2)

Because hypothermia has been shown to be an independent variable associated with mortality in groups of extreme preterm babies, it can now be argued that it would be unethical to show the improved outcomes using plastic bags by a further multicentred randomised trial, given that "conventionally" managed infants would probably experience an episode of preventable hypothermia in the early minutes of life, and such hypothermia has been shown to be independently associated with death. Clinicians, however, must be vigilant that, in achieving a mean temperature of $37^{\circ} \mathrm{C}$ on admission to the neonatal unit, they are not causing some infants to be hyperthermic. Is the latter intrinsically dangerous? Preterm infants are not immune from hypoxia/ ischaemia, and therefore theoretically there may be an increased risk of neurological damage following hyperthermia in some infants. ${ }^{14}{ }^{15}$ Caution should guide clinicians to monitor very closely not only the temperature range of preterm neonates on arrival at the neonatal unit, but also the minute by minute temperature of these infants in the subsequent hour while they stabilise in an incubator or under a radiant heat warmer. Until further evidence becomes available, it seems prudent to aim to prevent hypothermia, which clearly is associated with increased mortality in preterm infants, but also to strive to avoid hyperthermia, which may prove detrimental to infants once long term follow up studies have been completed.

\section{VAGARIES OF DECISION MAKING}

How does a clinician respond to the charge that he has chosen not to implement selective cooling in a predefined subgroup which has been subject to close scrutiny ${ }^{1}$ and yet has been persuaded to put extreme preterm infants into plastic bags without knowledge of long term outcomes? The answer is one of practicalities and a desire to do no harm. Selective head cooling requires expertise in neuroradiology and neurophysiology and training in the use of equipment currently unfamiliar to most neonatologists. Furthermore even those enthusiastic about head cooling recognise that it is not yet possible fully to define the precise relation between electrophysiology and secondary energy failure in brain cells. In addition, more needs to be discovered about the optimal cooling temperature that provides maximal protection for all the areas of the brain. The cautious clinician needs to be confident that the exciting preliminary findings of Gluckman and coworkers would be reproducible in other neonatal units for the benefit of this preselected subgroup of asphyxiated infants.

By contrast, it has been clearly shown that hypothermia is dangerous for the extreme preterm infant, and, by means of a simple plastic bag, avoidance of neonatal hypothermia is readily within the grasp of all meticulous clinicians. It seems prudent therefore to avoid the cold pathway, which is known to be dangerous, and choose a warmer route, still with a wary eye on new perils that may emerge.

\section{SUMMARY}

In this review, I have avoided the temptation to list the many changes in my practice in the last five years and the multiple influences that have brought about these changes. I have unashamedly concentrated on one subject. Important research is being carried out in the area of temperature control in two important groups. My only certainty is that an article on this subject in another five years will look very different.

Competing interests: none declared 


\section{REFERENCES}

1 Gluckman PD, Wyatt JS, Azzopardi D, et al. Selective head cooling with mild systemic hypothermia after neonatal encephalopathy: multicentred randomised trial. Lancet 2005;365:667-70.

2 Shankaran S, Laptook AR, Ehrenkrantz RA, et al. Whole body hypothermia for hypoxic-ischemic encephalopathy (HIE). Proceedings of Hot Topics in Neonatology 2004:151. Ross Laboratories.

3 Eicher DJ, Wagner CL, Katikaneni LP, et al. Moderate hypothermia in neonatal encephalopathy; efficacy outcomes. Pediatr Neurol 2005;32:11-17.

4 Eicher DJ, Wagner CL, Katikaneni LP, et al. Moderate hypothermia in neonatal encephalopathy: safety outcomes. Pediatr Neurol 2005;32:18-24.

5 Costeloe K, Hennessy E, Gibson AT, et al. The EPICure study: outcomes to discharge from hospital for infants born at the threshold of viability. Pediatrics 2000; 106:659-71.

6 Oxley C, Lyon AJ. Temperature on admission to the neonatal unit: a risk factor or just a reflection of condition at birth? Pediatr Res 2001;49:287.

7 Vohra S, Frent G, Campbell V, et al. Effect of polyethylene occlusive skin wrapping on heat loss in very low birth weight infants at delivery: a randomised trial. J Pediatr 1999;134:547-51.

8 Besch NJ, Perlstein PH, Edwards NK, et al. The transparent baby bag: a shield against heat loss. N Engl J Med 1971;284:121-4.
9 British Association of Perinatal Medicine/Royal College of Physicians. Development of audit measures and guidelines for good practice in the management of neonatal respiratory distress syndrome. Report of joint Working Group of British Association of Perinatal Medicine and the Research Unit of the Royal College of Physicians. Arch Dis Child 1992;67:1221-7.

10 CESDI Project 27/28. www.cemach.org.uk/publications/p2728/ mainreport.pdf

11 Lyon AJ, Stenson BJ. Cold comfort for babies. Arch Dis Child Fetal Neonatal Ed 2004;89:F93-4.

12 Meyer MP. Swaddling and heat loss. Arch Dis child Fetal Neonatal Ed 2003;88:F256.

13 Vohra S, Roberts RS, Zhang B, et al. Heat loss prevention (HeLP) in the delivery room: a randomised controlled trial of polyethylene occlusive skin wrapping in very preterm infants. J Pediatr 2004; 145:750-3.

14 Newton T, Watkinson M. Preventing hypothermia at birth in preterm babies: at a cost of overheating some? Arch Dis Child Fetal Neonatal Ed 2003;88:F256.

15 Gunn AJ, Bennet L. Is temperature important in delivery room resuscitation? Semin Neonatol $2001 ; 6: 241-9$.

\section{Call for papers}

11 th European Forum on Quality Improvement in Health Care

26-28 April 2006, Prague, Czech Republic

Deadline 30 September 2005.

For further information and to submit online go to: www.quality.bmipg.com 